InnOvaciOnes de NegOciOs 16(32): 202-231

(C) 2019 UANL, Impreso en México (ISSN: 2007-1191)

Recepción: 26 Mayo 2019 Aceptación: 3 Junio 2019

\title{
La imagen de la tienda en la satisfacción del cliente a través de la comunicación de boca a boca (The store image in customer satisfaction through word of mouth communication)
}

\author{
Rodolfo Treviño, Sergio Guerra, Eloísa Treviño \\ Universidad Autónoma de Nuevo León \& Universidad de Monterrey \\ rtrevino@hotmail.com, sagm52@hotmail.com, \\ ma.trevino@udem.edu
}

\begin{abstract}
Knowing the costumer perception through the store image is important to maintain a positive image in the consumer mind and a competitive advantage to remain in the market. The store image creates a satisfaction which leads to a positive word of mouth recommendation, being this important to retain customers and attract new ones. The literature review on these research topics is significant, given that the human being is changing in his way of thinking; therefore it is important that the stores have a constant monitoring to stay in the costumer taste. The objective of the study is to identify the elements that intervene in the store image in customer satisfaction through word of mouth communication. The methodology used is the literature review through databases with content of empirical studies. The results determine that the store image leads to customer
\end{abstract}

\section{La imagen de la tienda en la satisfacción del cliente}


satisfaction through word of mouth communication, as well as the elements that intervene in each of the categories forming the constructs for quantitative research.

Key words: Customer perception, store recommendation, costumer expectation.

JEL: M3, M31.

Resumen: Conocer la percepción del cliente a través de la imagen de la empresa es importante para mantener una imagen positiva en la mente del consumidor y una ventaja competitiva para permanecer en el mercado. La imagen de la tienda crea una satisfacción lo que conduce a una recomendación positiva de boca a boca, siendo esta importante para retener clientes y atraer nuevos. La revisión de la literatura en estos temas de investigación es significativa, dado que el ser humano es cambiante en su forma de pensar; por lo tanto es importante que las tiendas tengan un monitoreo constante para mantenerse en el gusto del cliente. El objetivo del estudio se encamina a identificar los elementos que intervienen en la imagen de la tienda en la satisfacción del cliente a través de la comunicación de boca a boca. La metodología utilizada es la revisión de la literatura a través de bases de datos con contenido de estudios empíricos. Los resultados determinan que la imagen de la tienda lleva a una

Treviño, R., Guerra, S., \& Treviño, E. 
satisfacción del cliente a través de una comunicación de boca a boca, así mismo se encontró los elementos que intervienen en cada una de las categorías formando los constructos para investigaciones cuantitativas.

Palabras clave: Percepción del cliente, recomendación de la tienda, expectativa del cliente.

\section{Introducción}

Según el Instituto Nacional de Estadística y Geografía (INEGI, 2009) el comercio donde se pueden adquirir productos; dentro de esta actividad las tiendas se encargan de acercar los productos a los clientes, la cual se exhibe en mostradores y vitrinas (INEGI, 2009). Conocer la percepción de la imagen de la tienda entre los clientes es de importancia para el posicionamiento de la tienda en su mercado meta. Mantenerse en la mente del consumidor es un objetivo clave, por lo que utilizar la imagen de la tienda para tal objetivo es un sello que lo distingue de las demás tiendas, que lo hace ver diferente y único, donde a través del tiempo esa imagen llega a ser considerada como confiable, en otras palabras seria.

\section{La imagen de la tienda en la satisfacción del cliente}


Para crear una imagen propia, la tienda desarrolla entre otras cosas: logotipos, slogan, marca, colores, etc. propios de la tienda que son retenidos en la mente del consumidor, creando una percepción única de la misma.

Por lo general, las empresas en todo el mundo invierten grandes cantidades de dinero en inversión publicitaria para mantener una imagen positiva en la mente del consumidor, a través de la televisión, el periódico o los medios digitales, por ejemplo Estados Unidos de América invirtió en el 2014, \$567 millones de dólares per cápita de inversión media anual, seguidos de Australia con $\$ 486$ y Noruega con $\$ 472$; México invirtió un total de $\$ 54$ muy por debajo de países como Japón, Canadá, Reino Unido o Brasil (Giménez, 2015) y lo anterior incluye a las tiendas, esto permite observar cómo las empresas a nivel mundial van dándose a conocer a través de la publicidad para crear una percepción positiva en el cliente acerca de la imagen de la tienda.

Por otra parte, la satisfacción del cliente puede ser una referencia para conocer si las tiendas cumplen 0 no con las expectativas del cliente (Rashed, Rifath, Begum y Bhuiyea, 2018; Yildiz, 2017). La American

Treviño, R., Guerra, S., \& Treviño, E. 
Marketing Association (2019) la define como una medida para conocer si los productos y/o servicios cumplen con las expectativas del cliente así, cumpliendo con las expectativas del cliente se espera que ellos mismos recomienden la tienda a otros futuros clientes, porque un cliente satisfecho es ideal para una comunicación positiva de boca a boca (Thomas, 2013), por lo que es importante conocer cómo influye la satisfacción del cliente con la comunicación de boca a boca (Tripathi, 2017), para conocer qué tipo de estrategia es la más adecuada y utilizar este medio para beneficio de la tienda.

Aunque no todas las tiendas están en condiciones de invertir en publicidad, el empresario tiene la opción de la comunicación de boca a boca para darse a conocer a los clientes, siendo esta una forma de publicidad que no le cuesta a la tienda obteniendo beneficios en la satisfacción del cliente (Lang y Hyde, 2013; Hamza, 2013), de esta forma se va colocando en la mente del consumidor una percepción positiva de la tienda y se va fortaleciendo económicamente para preparar campañas de publicidad que alcancen a un mayor número de clientes. La recomendación positiva de

\section{La imagen de la tienda en la satisfacción del cliente}


boca a boca evidencia una satisfacción del cliente (Burnham y Leary, 2018).

La información presentada permite considerar la importancia de la imagen de la tienda, donde si se tiene capital se invierten grandes cantidades económicas en ello, sin embargo existen tiendas con poco capital para invertir en su imagen a través de los medios masivos, por lo que una opción sin costo y con grandes beneficios es la comunicación positiva de boca a boca, la cual permite a la tienda retener a los clientes satisfechos y atraer a los nuevos, mientras se fortalece y hace inversiones económicas en su imagen.

A lo anterior surge la siguiente pregunta de investigación: ¿Cuáles son los elementos que intervienen en la imagen de la tienda para la satisfacción del cliente a través de la comunicación de boca a boca?, por lo que lleva al siguiente objetivo: Identificar los elementos que intervienen en la imagen en la tienda para la satisfacción del cliente a través de la comunicación de boca a boca.

Treviño, R., Guerra, S., \& Treviño, E. 
Revisión de la literatura

a) Imagen de la tienda

La imagen de la tienda es la forma en que el cliente la percibe al momento, la American Marketing Association, (2018) la define como el total de lo que los clientes piensan sobre una tienda en particular. Las definiciones de imagen de la tienda son múltiples, siendo en su conjunto mantener una imagen positiva en la mente de consumidor para mantener la supervivencia de la tienda en el tiempo. Teniendo en cuenta que lo importante para una tienda es mantener la satisfacción del cliente, la imagen es uno de los puntos más relevantes que deben cuidarse para lograr ese objetivo.

Los clientes se sienten atraídos a comprar cuando perciben una imagen positiva de la tienda (Chan y Chan, 2008) y a la vez la percepción de los clientes en la imagen de la tienda se ve afectada por los atributos de la misma (Žemgulienè, 2013). Existen diferentes atributos percibidos por los clientes que son tomados en cuenta en el momento de elegir una tienda y que repercuten en la imagen de la misma, entre ellos está la ubicación de la

\section{La imagen de la tienda en la satisfacción del cliente}


tienda, variedad de productos, comunicación de la tienda, precio, ambiente y servicio de la tienda (Deka, 2018). Una imagen de tienda fuerte tiene relación con la intención de recompra (Chang y Wang, 2014) y es un medio para diferenciarse de sus competidores (Champion, Hunt y Hunt, 2010). Diferentes criterios son utilizados por los clientes en el momento de realizar las compras, alguna tiendas utilizan su marca propia que los diferencian en cuanto a surtido e imagen de la tienda (Nies y Natter, 2012) en comparación con otras del mismo ramo. La imagen de la tienda está respaldada por el nombre de la tienda y la calidad de sus productos (Grewal, Krishnan, Baker y Borin, 1998) lo que lleva a una satisfacción del cliente.

\section{b) Satisfacción del cliente}

La satisfacción del cliente es primordial para la sobrevivencia de la tienda, esta se encuentra influenciada por las expectativas del cliente (Ara, 2016), es decir aquello que el cliente espera de la tienda. Un cliente queda satisfecho por la organización interna (Ltifi, Hikkerova y Gharbi, 2016) de la tienda cuando su estancia

\section{Treviño, R., Guerra, S., \& Treviño, E.}


en ella se torna placentera a través de un conjunto de factores internos, que siendo independientes se integran en un todo. Sin embargo, el entorno de la tienda esto es, aquellos factores externos que la tienda no puede controlar influyen también en la percepción del cliente (Mann y Jha, 2013) para su propia satisfacción. Las tiendas por lo general observan las motivaciones y el comportamiento de compra para entender la relación de estos con la satisfacción del cliente (Jack y Powers, 2013).

Por otra parte, diversos factores afectan la percepción del cliente que lleva a la satisfacción del cliente, entre estos están el precio, el entorno físico (Okursoy y Turan, 2014), la calidad del servicio (Okursoy y Turan, 2014; Tanwar, 2013; Hutcheson y Moutinho, 1998) y el valor del dinero (Hutcheson y Moutinho, 1998). Los clientes también evalúan los efectos visuales de la tienda desempeñando un factor importante en la intención de compra (Vieira, 2010), además el factor emocional y las instalaciones son clave en la satisfacción del cliente y aún más en la retención del mismo (Dastane y Fazlin, 2017). Asimismo, es de relevancia las expresiones de

\section{La imagen de la tienda en la satisfacción del cliente}


cortesía para lograr la satisfacción del cliente (Koermer, 2005). Un vendedor debe hacer que el comprador se sienta confortable, escucharlo y así recomendar los productos y servicios que tiene la tienda (Meyer, Barnes y Friend, 2017). Cuando el cliente considera que la tienda da explicaciones a los clientes se logra una mejor percepción, manteniendo así no solo la satisfacción sino también la lealtad del cliente (Lin, 2008). Otro factor importante es la calidad percibida con impacto positivo cuando las marcas vienen de países considerados con buena imagen por los clientes (Wu, 2011).

Los clientes satisfechos cuando desarrollan lealtad a la tienda la recomiendan a través de una comunicación de boca a boca siendo una manera de conseguir más clientes (Yildiz y Tehci, 2014), por lo que la satisfacción ejerce una relación importante con la comunicación de boca a boca (Arslanagic-Kalajdzic, Pestek y Mijanovic, 2014).

Treviño, R., Guerra, S., \& Treviño, E. 


\section{c) Comunicación de boca a boca}

Cuando el cliente crea sus expectativas de experiencias pasadas y lo da a conocer a otros, en la generalidad de las ocasiones lo realiza a través de la comunicación de boca a boca (Kannadasan y Aravazhi, 2015). La satisfacción del cliente tiene una influencia positiva en este tipo de comunicación (Lee, 2016), es decir existe un relación entre la satisfacción y la comunicación de boca a boca donde las tiendas observan la satisfacción del cliente para trazar una comunicación de boca a boca positiva (Tripathi, 2017) que es aprovechada por las tiendas en el área de marketing (Wangenheim y Bayón, 2007). Se ha observado que las intenciones de recompra y la comunicación de boca a boca están influenciadas con la satisfacción del cliente (Dos Santos y Von Der Heyde Fernandes, 2008; Yi y Gong, 2008). Si bien las tiendas desarrollan estrategias basándose en la satisfacción del cliente (Ranaweera y Prabhu, 2003) que mejor incentivo para ellas que observar que la satisfacción tiene una relación positiva

\section{La imagen de la tienda en la satisfacción del cliente}


con la comunicación de boca a boca (De Matos y Rossi, 2008).

La comunicación de boca a boca se desarrolla en forma lenta, pero añade un valor a largo plazo para la tienda (Villanueva, Yoo y Hanssens, 2008), siendo así que la misma reputación favorable de la tienda y la satisfacción del cliente pronostican una relación positiva de boca a boca (Hong y Yang, 2009).

Comprender las causas de satisfacción que intervienen en los clientes son necesarias para una comunicación positiva de boca a boca (Mende, Thompson y Coenen, 2015). Algunos factores que se han estudiado y que influyen para una comunicación positiva de boca a boca son la eficiencia del personal y la satisfacción del precio (Lymperopoulos y Chaniotakis, 2008). Las tiendas están manejando una relación vendedor-cliente para establecer lazos de lealtad con la tienda teniendo efectos significativos en este tipo de comunicación (Reynolds y Arnold, 2000). Es importante en la percepción de los clientes que se sientan escuchados por los vendedores para establecer de manera positiva la confianza, satisfacción, comunicación

Treviño, R., Guerra, S., \& Treviño, E. 
positiva, intenciones de compra, etc. (Bergeron y Laroche, 2009), de hecho los vendedores deben de asignar un mayor empeño en establecer estrategias en la comunicación de boca a boca (Shields, 2006). La percepción del cliente en la calidad del servicio es otro factor a considerar, juega un papel importante a través de la confianza para generar una comunicación positiva de boca a boca (Mittal y Gera, 2012; Yingzi, Goedegebuure y Van der Heijden, 2006).

Tener una ventaja competitiva es necesaria para competir en un mercado cada vez más competitivo y se logra ganándose la confianza del cliente a través de estándares éticos. La forma en que es percibida la justicia influye en la confianza y en el compromiso que lleva a su vez a este tipo de comunicación (Wang y Chang, 2013; Şafakli, 2010). La satisfacción del cliente es necesaria para mantener la ventaja competitiva en el mercado consiguiendo a su vez una comunicación positiva de boca a boca (Arambewela, Hall y Zuhair, 2005).

\section{La imagen de la tienda en la satisfacción del cliente}




\section{Metodología}

El estudio identifica los elementos que intervienen en cada una de las categorías llamadas: imagen de la tienda, satisfacción del cliente y comunicación de boca a boca con el fin de establecer, con base en la literatura revisada, la relación conceptual entre ellas. Para llevarlo a cabo fue necesario seguir las siguientes etapas:

Etapa 1: Búsqueda de información

La búsqueda de información se realizó a través de bases de datos de información científica.

Etapa 2: Análisis de la información

Para el análisis de la información se utilizó el análisis de contenido a través de codificación abierta. El procesamiento de datos utilizado en la investigación es la lista de términos y palabras clave, donde la elección de las palabras expuestas ayuda a la identificación de las categorías y subcategorías encontradas.

Treviño, R., Guerra, S., \& Treviño, E. 


\section{Etapa 3: Conceptualización de las categorías}

Una vez que se tuvo analizada la información se procedió, de acuerdo a la literatura revisada, a la conceptualización de imagen de la empresa, satisfacción del cliente y comunicación de boca a boca como se observa en la tabla 1.

Tabla 1. Conceptualización de categorías

\begin{tabular}{ll}
\hline Categoría & Conceptualización \\
\hline $\begin{array}{l}\text { Imagen de la } \\
\text { tienda }\end{array}$ & $\begin{array}{l}\text { Serie de elementos percibidos por el cliente } \\
\text { que crean una actitud favorable o } \\
\text { desfavorable hacia las tiendas }\end{array}$ \\
$\begin{array}{l}\text { Satisfacción del } \\
\text { cliente }\end{array}$ & $\begin{array}{l}\text { Resultado de la percepción del cliente con } \\
\text { respecto a la imagen de la tienda }\end{array}$ \\
$\begin{array}{l}\text { Comunicación de } \\
\text { boca a boca }\end{array}$ & $\begin{array}{l}\text { Transmisión de la información del cliente } \\
\text { con respecto a la satisfacción percibida }\end{array}$
\end{tabular}

Fuente: Elaboración propia

Etapa 4: Obtención del resultado

Gracias a la conceptualización se obtienen las subcategorías encontradas para cada categoría. La tabla

\section{La imagen de la tienda en la satisfacción del cliente}


2 muestra las categorías y subcategorías que influyen en la imagen de la empresa, satisfacción del cliente y comunicación de boca a boca.

Tabla 2. Conceptualización de subcategorías

\begin{tabular}{|c|c|c|}
\hline Categoría & Subcategoría & Autores \\
\hline $\begin{array}{l}\text { Imagen de } \\
\text { la tienda }\end{array}$ & $\begin{array}{l}\text { Ubicación de } \\
\text { la tienda } \\
\text { Variedad de } \\
\text { productos } \\
\text { Comunicación } \\
\text { de la tienda } \\
\text { Precio } \\
\text { satisfactorio } \\
\text { Ambiente en } \\
\text { la tienda } \\
\text { Calidad del } \\
\text { servicio } \\
\text { Marca propia } \\
\text { Surtido de la } \\
\text { tienda } \\
\text { Nombre de la } \\
\text { tienda } \\
\text { Calidad de los } \\
\text { productos }\end{array}$ & $\begin{array}{l}\text { Deka, } 2018 \\
\text { Deka, } 2018 \\
\text { Deka, } 2018 \\
\text { Deka, } 2018 \\
\text { Deka, } 2018 \\
\text { Deka, } 2018 \\
\text { Nies y Natter, } 2012 \\
\text { Nies y Natter, } 2012 \\
\text { Grewal, Krishnan, Baker y Borin, } \\
1998 \\
\text { Grewal, Krishnan, Baker y Borin, } \\
1998\end{array}$ \\
\hline
\end{tabular}

Treviño, R., Guerra, S., \& Treviño, E. 


\begin{tabular}{|c|c|c|}
\hline \multirow{10}{*}{$\begin{array}{l}\text { Satisfacci } \\
\text { ón del } \\
\text { cliente }\end{array}$} & $\begin{array}{l}\text { Expectativas } \\
\text { del cliente }\end{array}$ & Ara, 2016 \\
\hline & $\begin{array}{l}\text { Organización } \\
\text { interna de la } \\
\text { tienda }\end{array}$ & Ltifi, Hikkerova y Gharbi, 2016 \\
\hline & $\begin{array}{l}\text { Sentirse } \\
\text { confortable en } \\
\text { la tienda }\end{array}$ & Meyer, Barnes y Friend, 2017 \\
\hline & $\begin{array}{l}\text { Precio } \\
\text { satisfactorio }\end{array}$ & Okursoy y Turan, 2014 \\
\hline & $\begin{array}{l}\text { Calidad del } \\
\text { servicio }\end{array}$ & $\begin{array}{l}\text { Okursoy y Turan, 2014; Tanwar, } \\
\text { 2013; Hutcheson y Moutinho, } \\
1998\end{array}$ \\
\hline & $\begin{array}{l}\text { Efectos } \\
\text { visuales de la } \\
\text { tienda }\end{array}$ & Vieira, 2010 \\
\hline & $\begin{array}{l}\text { Instalaciones } \\
\text { satisfactorias }\end{array}$ & Dastane y Fazlin, 2017 \\
\hline & $\begin{array}{l}\text { Expresiones } \\
\text { de cortesía }\end{array}$ & Koermer, 2005 \\
\hline & $\begin{array}{l}\text { Escuchar al } \\
\text { cliente }\end{array}$ & Meyer, Barnes y Friend, 2017 \\
\hline & $\begin{array}{l}\text { Proporcionar } \\
\text { explicaciones } \\
\text { al cliente }\end{array}$ & Lin, 2008 \\
\hline
\end{tabular}

\section{La imagen de la tienda en la satisfacción del cliente}




\begin{tabular}{|c|c|c|}
\hline & $\begin{array}{l}\text { Marcas } \\
\text { extranjeras en } \\
\text { la tienda }\end{array}$ & Wu, 2011 \\
\hline \multirow[t]{8}{*}{$\begin{array}{l}\text { Comunica } \\
\text { ción de } \\
\text { boca a } \\
\text { boca }\end{array}$} & $\begin{array}{l}\text { Confianza en } \\
\text { la tienda }\end{array}$ & $\begin{array}{l}\text { Bergeron y Laroche, 2009; Mittal } \\
\text { y Gera, 2012; Yingzi, } \\
\text { Goedegebuure y Van der } \\
\text { Heijden, } 2006\end{array}$ \\
\hline & $\begin{array}{l}\text { Creación de } \\
\text { expectativas } \\
\text { pasadas }\end{array}$ & Kannadasan y Aravazhi, 2015 \\
\hline & $\begin{array}{l}\text { Reputación de } \\
\text { la tienda }\end{array}$ & Hong y Yang, 2009 \\
\hline & $\begin{array}{l}\text { Eficiencia del } \\
\text { personal }\end{array}$ & $\begin{array}{l}\text { Lymperopoulos y Chaniotakis, } \\
2008\end{array}$ \\
\hline & $\begin{array}{l}\text { Precio } \\
\text { satisfactorio }\end{array}$ & $\begin{array}{l}\text { Lymperopoulos y Chaniotakis, } \\
2008\end{array}$ \\
\hline & $\begin{array}{l}\text { Experiencia } \\
\text { de relación } \\
\text { vendedor- } \\
\text { cliente }\end{array}$ & Reynolds y Arnold, 2000 \\
\hline & $\begin{array}{l}\text { Escuchar al } \\
\text { cliente }\end{array}$ & Bergeron y Laroche, 2009 \\
\hline & $\begin{array}{l}\text { Intención de } \\
\text { compra }\end{array}$ & Bergeron y Laroche, 2009 \\
\hline
\end{tabular}

Treviño, R., Guerra, S., \& Treviño, E. 
Calidad del Mittal y Gera, 2012; Yingzi, servicio Goedegebuure y Van der Heijden, 2006

Fuente: Elaboración propia en base a literatura revisada

Una vez obtenidas las subcategorías en la imagen de la tienda, la satisfacción del cliente y la comunicación de boca a boca, se obtiene el siguiente modelo conceptual. La figura 1 muestra el modelo conceptual que relaciona en forma lineal las categorías estudiadas, llegando al resultado que una imagen positiva de la tienda lleva a una satisfacción de cliente dando como resultado una comunicación positiva de boca a boca.

La imagen de la tienda en la satisfacción del cliente 
Figura 1. Modelo de relación entre las categorías y subcategorías

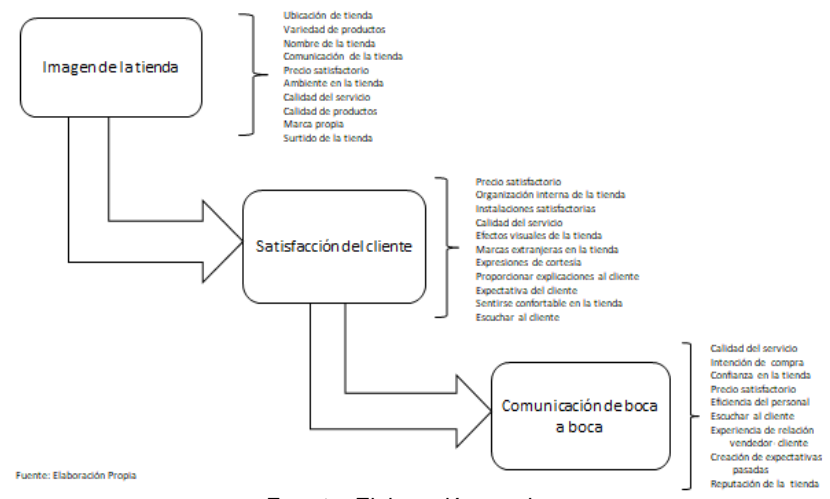

Fuente: Elaboración propia

\section{Conclusiones}

La revisión de la literatura sugiere que la imagen de la tienda, la satisfacción del cliente y la recomendación de boca a boca sigue siendo tema de investigación. Las tiendas enfrentan hoy en día una competencia fuerte para permanecer en el mercado. Conocer la percepción del cliente es necesario para obtener una ventaja competitiva

Treviño, R., Guerra, S., \& Treviño, E. 
que les permita mantenerse vigente a través de la satisfacción del cliente. La imagen de la tienda crea una satisfacción del cliente que a su vez conduce a una recomendación de boca a boca, siendo éste un medio para atraer clientes a las tiendas. Los hallazgos señalan que al poseer 0 administrar una tienda ciertos elementos como la calidad del servicio, el precio satisfactorio entre otros, crean una imagen positiva de la tienda, lo que a su vez genera una satisfacción que lleva a tener una posición competitiva respecto a sus competidores. La recomendación a través de la comunicación de boca a boca es importante; si una tienda es del agrado de los clientes tendrá un efecto positivo a través de un medio que no tiene costo para la misma, trayéndole grandes beneficios con la llegada de más clientes, necesarios para su supervivencia y desarrollo.

Al conocer las categorías y subcategorías reflejadas en los estudios de investigación revisados pone de manifiesto los elementos a considerar en la decisión y las estrategias empresariales. Cada una de estas categorías: imagen de la tienda, satisfacción del cliente y comunicación de boca a boca, contiene una serie de

\section{La imagen de la tienda en la satisfacción del cliente}


elementos que si los llevamos al ámbito cuantitativo forman sus constructos. Los resultados del estudio ponen de manifiesto los constructos que a través de diversos estudios se han encontrado para cada una de las categorías

Líneas futuras de investigación

Las futuras líneas de investigación se encaminan hacia la investigación no experimental, paramétrica utilizando la regresión lineal como método estadístico con el fin de determinar la causa y el efecto entre la variable Imagen de la tienda considerándola variable independiente y la variable comunicación boca a boca examinándola como variable dependiente, teniendo una variable mediadora llamada satisfacción del cliente.

\section{Referencias}

American Marketing Association (2019). Customer Satisfaction. Recuperado

https://www.ama.org/resources/Pages/Dictionary.aspx?dL etter $=C$

Treviño, R., Guerra, S., \& Treviño, E. 
American Marketing Association (2018). Store Image. Recuperado de https://www.ama.org/resources/Pages/Dictionary.aspx?dL etter $=S$

Ara, A. (2016). Guest Satisfaction in Hotels of Kashmir: The Perception of Foreign Tourists. International Journal of Research in Commerce \& Management, 7(4), 88-93.

Arambewela, R. R., Hall, J. J. y Zuhair, S. S. (2005). Postgraduate International Students from Asia: Factors Influencing Satisfaction. Journal of Marketing for Higher Education, 15(2), 105-127. doi: 10.1300/J050v15n02_05

Arslanagic-Kalajdzic, M., Pestek, A. y Mijanovic, J. (2014). Influence of Customer Satisfaction and Loyalty on Word of Mouth. Conference Proceedings: International Conference of The Faculty of Economics Sarajevo (ICES), 222

Bergeron, J. y Laroche, M. (2009). The effects of perceived salesperson listening effectiveness in the financial industry. Journal of Financial Services Marketing, 14(1), 6-25. doi:10.1057/fsm.2009.1

Burnham, T. A. y Leary, R. B. (2018). Word of Mouth Opportunity: Why Recommendation Likelihood Overestimates Positive Word of Mouth. Journal of Marketing Theory \& Practice, 26(4), 368-389

Champion, J. J., Hunt, J. H. y Hunt, T. H. (2010). The Effect of Retail Store Image on Student Perceptions of Merchandise Quality and Willingness to Buy. American Journal of Business Research, 3(1), 17-32

\section{La imagen de la tienda en la satisfacción del cliente}


Chan, J. Y. y Chan, P. L. (2008). Merchandise Display affects Store Image. Advances in Consumer Research - European Conference Proceedings, 8408-414

Chang, S. y Wang, K. (2014). Investigating the Antecedents and Consequences of an Overall Store Price Image in Retail Settings. Journal of Marketing Theory \& Practice, 22(3), 299-314. doi:10.2753/MTP1069-6679220305

Dastane, O. y Fazlin, I. (2017). Reinvestigating Key Factors of Customer Satisfaction Affecting Customer Retention for Fast Food Industry. International Journal of Management, Accounting \& Economics, 4(4), 379-400

De Matos, C. A. y Rossi, C. V. (2008). Word-of-mouth communications in marketing: a meta-analytic review of the antecedents and moderators. Journal of the Academy of Marketing Science, 36(4), 578-596

Deka, P. K. (2018). Factors Influencing Consumers' Choice of Retail Store Format in Assam, India. IUP Journal of Marketing Management, 17(2), 22-37

Dos Santos, C. P. y Von Der Heyde Fernandes, D. (2008). A Recuperação de Serviços como Ferramenta de Relacionamento e Seu Impacto na Confiança e Lealdade Dos Clientes. Revista De Administração De Empresas, 48(1), 10-24

Giménez, A. 2015. Las Empresas Que Más Invierten En Marketing Del Mundo. Recuperado de https://www.codigonuevo.com/sociedad/empresasinvierten-marketing-mundo

Treviño, R., Guerra, S., \& Treviño, E. 
Grewal, D., Krishnan, R., Baker, J. y Borin, N. (1998). The Effects of Store Name, Brand Name and Price Discounts on Consumers' Evaluations and Purchase Intentions. Journal of Retailing, 74(3), 331-352

Hong, S. Y. y Yang, S. (2009). Effects of Reputation, Relational Satisfaction, and Customer-Company Identification on Positive Word-of-Mouth Intentions. Journal of Public Relations Research, 21(4), 381-403. doi: 10.1080/10627260902966433

Hutcheson, G. D. y Moutinho, L. (1998). Measuring Preferred Store Satisfaction Using Consumer Choice Criteria as a Mediating Factor. Journal of Marketing Management, 14(7), 705-720

Jack, E. P. y Powers, T. L. (2013). Shopping Behaviour and Satisfaction Outcomes. Journal of Marketing Management, 29(13-14), 1609-1630. doi:10.1080/0267257X.2013.798678

Kannadasan, S. y Aravazhi, D. (2015). Customer Satisfaction in Marketing. International Journal of Research in Commerce \& Management, 6(8), 33-34

Koermer, C. D. (2005). Service Provider Type as a Predictor of The Relationship Between Sociality and Customer Satisfaction. Journal of Business Communication, 42(3), 247-264. doi: 10.1177/0021943605277068

Lang, B. y Hyde, K. F. (2013). Word of Mouth: What We Know and What We Have yet to Learn. Journal of Consumer Satisfaction, Dissatisfaction \& Complaining Behavior, 26, $1-18$

\section{La imagen de la tienda en la satisfacción del cliente}


Lee, Y. (2016). Relationship Quality and Its Causal Link to Service Value, Satisfaction, and Word-of-Mouth. Services Marketing Quarterly, 37(3), 171-184. doi:10.1080/15332969.2016.1184541

Lin, C. (2008). An evaluation of Explanation Effects on Consumer Perceptions. Social Behavior \& Personality: An International Journal, 36(3), 373-384

Ltifi, M., Hikkerova, L. y Gharbi, J. (2016). L'effet de la performance logistique en magasin de détail sur la satisfaction et le bonheur des consommateurs. Revue Des Sciences De Gestion, 278(279), 89-98

Lymperopoulos, C. y Chaniotakis, I. E. (2008). Price satisfaction and personnel efficiency as antecedents of overall satisfaction from consumer credit products and positive word of mouth. Journal of Financial Services Marketing, 13(1), 63-71. doi:10.1057/fsm.2008.6

Hamza, V. K. (2013). A Study on the Mediation Role of Customer Satisfaction on Customer Impulse and Involvement to Word of Mouth and Repurchase Intention. International Journal of Business Insights \& Transformation, 7(1), 62-67.

Instituto Nacional de Estadística y Geografía (2009). Comercio. Disponible en: http://cuentame.inegi.org.mx/economia/parque/comercio.ht $\mathrm{ml}$

Mann, P. W. y Jha, M. (2013). Impact of Various Situational Factors on "Store Environment, Merchandising and Consumer

Treviño, R., Guerra, S., \& Treviño, E. 
Behavior" -- A Study on Furniture Bazaar. Journal of Marketing \& Communication, 9(2), 29-37

Mende, M., Thompson, S. y Coenen, C. (2015). It's all relative: how customer-perceived competitive advantage influences referral intentions. Marketing Letters, 26(4), 661-678. doi:10.1007/s11002-014-9318-x

Meyer, T., Barnes, D. C. y Friend, S. B. (2017). The role of delight in driving repurchase intentions. Journal of Personal Selling \& Sales $\quad$ Management, 37(1), 61-71. doi:10.1080/08853134.2016.1272052

Mittal, S. y Gera, R. (2012). Relationship Between Service Quality Dimensions and Behavioural Intentions: An Sem Study of Public Sector Retail Banking Customers in India. Journal of Services Research, 12(2), 147-171

Nies, S. y Natter, M. (2012). Does Private Label Quality Influence Consumers' Decision on Where to Shop?. Psychology \& Marketing, 29(4), 279-292. doi:10.1002/mar.20521

Okursoy, A. y Turan, A. H. (2014). Açimlayici Faktör Analizi Ve Üniversite Yemekhanesinde Müşteri Memnuniyeti Üzerinde Etkili Olan Boyutlarin Belirlenmesi Üzerine Bir Uygulama. Dogus University Journal, 15(1), 65-78

Ranaweera, C. y Prabhu, J. (2003). On the relative importance of customer satisfaction and trust as determinants of customer retention and positive word of mouth. Journal of Targeting, Measurement \& Analysis for Marketing, 12(1), 82-90

Rashed, C. A. A., Rifath, J., Begum, R. y Bhuiyea, M. S. H. (2018). Investigation of Satisfaction Level of Customers and Hidden

\section{La imagen de la tienda en la satisfacción del cliente}


Factors for Selected Retail Store Shopping. Review of General Management, 27(1), 22-43

Reynolds, K. E. y Arnold, M. J. (2000). Customer Loyalty to the Salesperson and the Store: Examining Relationship Customers in an Upscale Retail Context. Journal of Personal Selling \& Sales Management, 20(2), 89-98

Şafakli, O. V. (2010). The Impact of Ethical Perceptions of Small and Medium Sized Enterprises (SMEs) on Their Bank Satisfaction and Subsequent Word of Mouth: Case of Northern Cyprus. Ege Academic Review, 10(1), 37-51

Shields, P. O. (2006). Customer Correspondence: Corporate Responses and Customer Reactions. Marketing Management Journal, 16(2), 155-170

Tanwar, S. (2013). Prioritizing Service Quality Attributes: A Study of Indian Apparel Retail Stores. Journal of Marketing \& Communication, 9(2), 52-61

Thomas, S. (2013). Linking customer loyalty to customer satisfaction and store image: a structural model for retail stores. Decision 40(1-2), 15-25

Tripathi, G. (2017). Customer Satisfaction and Word of Mouth Intentions: Testing the Mediating Effect of Customer Loyalty. Journal of Services Research, 17(2), 1-16

Vieira, V. (2010). Visual Aesthetics in Store Environment and its Moderating Role on Consumer Intention. Journal of Consumer Behaviour, 9(5), 364-380

Villanueva, J., Yoo, S. y Hanssens, D. M. (2008). The Impact of Marketing-Induced Versus Word-of-Mouth Customer

Treviño, R., Guerra, S., \& Treviño, E. 
Acquisition on Customer Equity Growth. Journal of Marketing Research, 45(1), 48-59. doi: 10.1509/jmkr.45.1.48

Wang, E. S., \& Chang, S. (2013). Creating Positive Word-of-Mouth Promotion through Service Recovery Strategies. Services Marketing Quarterly, 34(2), 103-114. doi:10.1080/15332969.2013.770661

Wangenheim, F. V. y Bayón, T. (2007). The chain from customer satisfaction via word-of-mouth referrals to new customer acquisition. Journal of the Academy of Marketing Science, 35(2), 233-249

Wu, P. S. (2011). Extrinsic Cue Effects on Consumers' Quality and Risk Perceptions of Private Label Brands. Marketing Review, 8(3), 385-404

Yi, Y. y Gong, T. (2008). The electronic service quality model: The moderating effect of customer self-efficacy. Psychology \& Marketing, 25(7), 587-601

Yildiz, E. (2017). Effects of Service Quality on Customer Satisfaction, Trust, Customer Loyalty and Word of Mouth: An Application on Cargo Companies in Gümüşhane. Global. Journal of Economics \& Business, 6(12), 81-88

Yildiz, S. y Tehci, A. (2014). Ağızdan Ağıza İletişimde Müşteri Tatmini ve Müşteri Sadakati ile Mağaza İmajı Boyutları: Ordu Ilinde Bir Uygulama. Journal of Graduate School of Social Sciences, 18(1), 441-460

Yingzi, X., Goedegebuure, R. y Van der Heijden, B. (2006). Customer Perception, Customer Satisfaction, and Customer Loyalty

\section{La imagen de la tienda en la satisfacción del cliente}


Within Chinese Securities Business: Towards a Mediation Model for Predicting Customer Behavior. Journal of Relationship Marketing, 5(4), 79-104. doi: 10.1300/J366v05n04_06

Žemgulienè, J. (2013). Relative importance of retail store image and consumers characteristics on the perception of value and willingness to pay a premium price. Regional Formation \& Development Studies, (9), 157-165

Treviño, R., Guerra, S., \& Treviño, E. 